\title{
Realization of fiber based laser Doppler vibrometer with serrodyne frequency shifting
}

\author{
Stijn Meersman, ${ }^{1}$ Yanlu Li, ${ }^{1, *}$ and Roel Baets ${ }^{1}$ \\ ${ }^{1}$ Photonics Group (INTEC), Ghent University-IMEC, Sint-Pietersnieuwstraat 41, 9000 \\ Gent, Belgium \\ *Corresponding author: Yanlu.Li@intec.ugent.be
}

\begin{abstract}
We demonstrate a laser Doppler vibrometer (LDV) based on a serrodyne frequency shifter (SFS). A proof-of-principle system is implemented on the basis of fiber-optic components but opens the way towards an ultra-compact integrated LDV-system on a silicon chip. With a low laser power of $50 \mu \mathrm{W}$, the serrodyne LDV was able to measure sub-micron vibrations with frequencies in the audio range. (c) 2010 Optical Society of America
\end{abstract}

OCIS codes: 000.0000, 999.9999.

\section{Introduction}

A laser Doppler vibrometer (LDV) is an optical sensor used for non-contact vibration measurements. According to the Doppler effect, a laser reflected by a moving surface will undergo a frequency shift of $f_{D}(t)$, which is proportional to the instantaneous velocity of the out-of-plane vibrations (vibrating in the direction of the laser beam) [1]. An LDV can recover the out-of-plane velocity by extracting the instantaneous frequency shift of the reflected signals. Nowadays, LDVs have been exploited in a wide variety of applications, such as acoustics [2], aerospace [3] and hearing research [4].

Most LDV devices make use of the heterodyne detection method because of its better sensitivity and higher reliability [5], and optical frequency shifters (OFSs) are required in this system. The reason to use OFSs is twofold: 1. it provides LDVs with the ability to measure the directions of vibrations [6]; 2. it shifts the frequency band away from the low frequency region which is quite noisy, and thus enhances the signal to noise ratio (SNR) of the obtained signals [1]. Bragg cells, which can realize a frequency shift by using the acousto-optic effect, are the most commonly used OFSs in free space heterodyne LDVs.

However, the flexibility of commercialized LDVs is still limited by the size of the equipment, and therefore there is an interest in miniaturized LDVs. Miniaturized LDVs could also 
have other advantages over bulk LDVs, such as low power consumption and low fabrication cost. Our proposal for a miniaturized LDV is based on CMOS technology, which can be a platform of different micro-photonic devices (e.g. waveguides, couplers, lasers and modulators) and make low cost mass production possible [7-11]. With this technology, an LDV can be fabricated on an ultra-compact silicon-on-insulator (SOI) chip with an area smaller than $1 \mathrm{~mm}^{2}$.

However, it is very challenging to realize an acoustooptic Bragg cell on SOI. The main reason relates to the difficulty of generating acoustic waves on the patterned SOI chips. Instead of the acousto-optic effect, we propose to use the serrodyne OFS (SFS) technique for generating a frequency shift. This method was developed and analyzed by Cumming [12], and later it was also applied to lasers in the telecommunication windows [13]. More details about it will be discussed in section 3 .

In this paper we demonstrate a proof-of-principle LDV based on a serrodyne frequency shifter. This proof-of-principle set-up is not yet an integrated implementation but is based on fiber components. With this setup we tried to measure vibrations in the audio frequency range. In the measurement, we also tried to limit our laser power as much as possible (down to $50 \mu \mathrm{W}$ ), so that we can estimate the power budget of this system.

In section 2, we will first discuss the principle of the heterodyne LDV. The idea of serrodyne OFS frequency shifting is proposed in section 3. We will then describe the fiber-based setup used to demonstrate the principle of serrodyne LDVs in section 4 . The measurement results on audio frequency vibrations are discussed in section 5 . The conclusion will be in the final part of this paper.

\section{Principle of heterodyne LDV}

A heterodyne LDV can be realized with a Mach-Zehnder interferometer (MZI) (shown in figure 1). A laser beam with a frequency $f_{0}$ is split by the optical coupler (SPLITTER in figure 1) into the reference and measurement signals. The reference signal is frequency shifted by an OFS and expressed as

$$
E_{r}(t)=E_{r} \exp \left[i 2 \pi\left(f_{0}+f_{\mathrm{OFS}}\right) t\right]
$$

where $E_{r}$ is the amplitude of the reference signal's electric field, and $f_{\mathrm{OFS}}$ is the constant frequency shift created by the OFS. The measurement signal is sent to a vibrating surface, and a part of its reflection is collected by the interferometer. Due to the movement of the surface, the measurement signal undergoes an instantaneous phase shift of $\phi_{D}(t)$. The collected signal can be written as

$$
E_{m}(t)=E_{m} \sqrt{\alpha} \exp \left\{i\left[2 \pi f_{0} t+\phi_{D}(t)\right]\right\}
$$




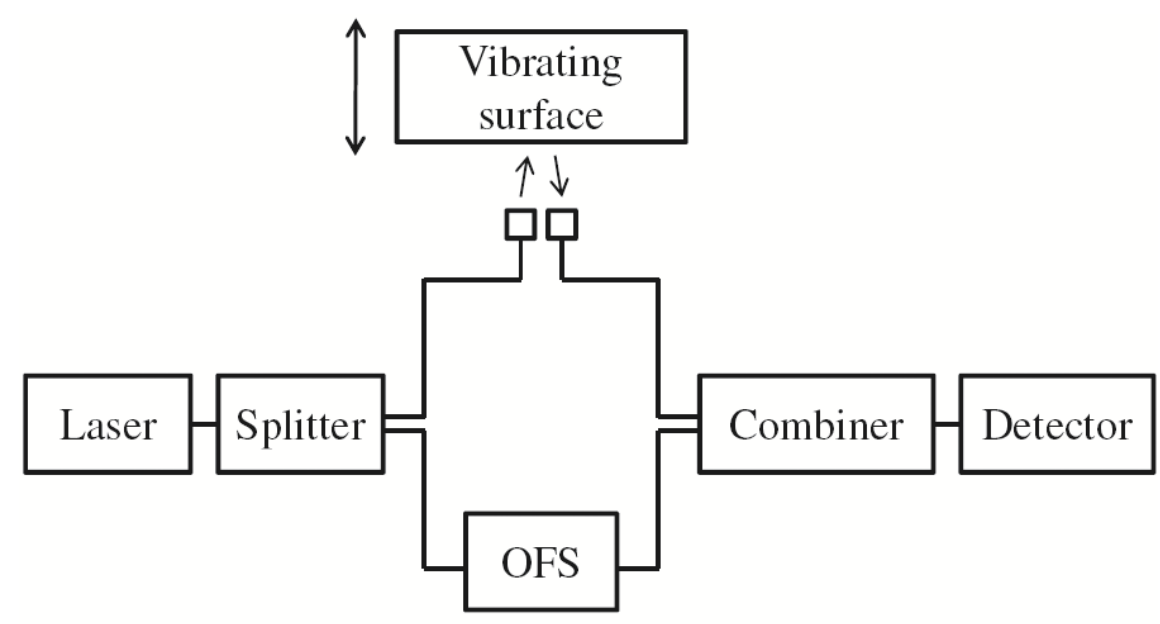

Fig. 1. The structure of a heterodyne LDV.

where $E_{m}$ is the amplitude of the measurement signal's electric field before it is sent to the vibrating surface, and $\alpha$ is the optical power loss due to the free space diffraction, scattering, and reflection loss of the signal. The Doppler shift is the derivative of the phase shift

$$
f_{D}(t)=\frac{d \phi_{D}(t)}{d t} .
$$

The reference and measurement signals are recombined in another optical coupler (COMBINER in figure 1), and the recombined signal is sent to a photo-detector. The resulting photo current can be expressed as

$$
\begin{aligned}
S_{o}(t) & \propto\left|E_{m}(t)+E_{r}(t)\right|^{2} \\
& =I_{r}+I_{m}+I_{h}(t),
\end{aligned}
$$

where $I_{r}=E_{r}^{2}, I_{m}=\alpha\left(E_{m}\right)^{2}$, and

$$
I_{h}(t)=2 E_{r} E_{m} \sqrt{\alpha} \cos \left[2 \pi f_{\mathrm{OFS}} t-\phi_{D}(t)\right] .
$$

Before sending the signal to the demodulator, we filter away the DC signals $I_{r}$ and $I_{m}$ since they are not useful in the demodulation. The demodulator finally receives a frequency modulated signal $S(t)$ which is proportional to $I_{h}(t)$. Later in the paper, we will omit the coefficient of $I_{h}(t)$, and assume that $S(t)=I_{h}(t)$.

We can do the demodulation digitally with the help of an analog to digital converter (ADC). The instantaneous phase shifts $\phi_{D}(t)$ can be recovered with the following procedure: 
1. creating two copies of the signal $S(t)$;

2. mixing them with two local oscillating signals $A(t)=\sin \left(2 \pi f_{\mathrm{OFS}} t\right)$ and $B(t)=$ $\cos \left(2 \pi f_{\mathrm{OFS}} t\right)$, and obtaining two mixture signals

$$
\begin{aligned}
& S_{A 0}(t)=E_{r} E_{m} \sqrt{\alpha}\left\{\sin \left[\phi_{D}(t)\right]+\sin \left[4 \pi f_{\mathrm{OFS}}-\phi_{D}(t)\right]\right\} \\
& S_{B 0}(t)=E_{r} E_{m} \sqrt{\alpha}\left\{\cos \left[\phi_{D}(t)\right]+\cos \left[4 \pi f_{\mathrm{OFS}}-\phi_{D}(t)\right]\right\}
\end{aligned}
$$

3. getting rid of high frequency components with low pass filters

$$
\begin{aligned}
& S_{A}(t)=E_{r} E_{m} \sqrt{\alpha} \sin \left[\phi_{D}(t)\right] \\
& S_{B}(t)=E_{r} E_{m} \sqrt{\alpha} \cos \left[\phi_{D}(t)\right]
\end{aligned}
$$

4. calculating the recovered phase shift by

$$
\phi_{D}(t)=\arctan \left(S_{A} / S_{B}\right)
$$

With the obtained phase shift $\phi_{D}(t)$, the instantaneous frequency shift $f_{D}(t)$ can be obtained from the equation (3).

\section{Serrodyne OFS}

To generate a single sideband frequency shift, we need an optical frequency shifter (OFS). Here we propose to use the serrodyne method. First, consider a coherent optical signal that passes through a phase modulator. When the phase modulator is driven by a periodic signal with period $=1 / f_{\mathrm{OFS}}$, many harmonic sidebands will appear in the signal's spectrum. In figure 2(a), the spectrum of a sinusoidal phase modulation is shown. In order to generate a pure frequency shift of $f_{\mathrm{OFS}}$, we need to suppress all the other sidebands except the 1st harmonic order. An ideal serrodyne OFS (SFS) can realize this effect, the corresponding spectrum after modulation is shown in figure 2(b). The SFS is accomplished by creating a specific sawtooth phase profile $\varphi(t)$ in the optical signal. The phase profile can be described in a piecewise linear function

$$
\varphi(t)=2 \pi\left[t f_{\mathrm{OFS}}-\text { floor }\left(t f_{\mathrm{OFS}}\right)\right]
$$

where $f_{\text {OFS }}$ is the optical frequency shift and floor $(t)$ is the floor function of $t$. This phase profile is also shown in figure 3 .

Since the duration of the $2 \pi$ phase jump is infinitely small, the optical field does not feel this jump. As a result, the described sawtooth phase profile has the same effect as a linear phase profile $2 \pi f_{\text {OFS }}$. Hence, the sawtooth phase modulation will create a same 


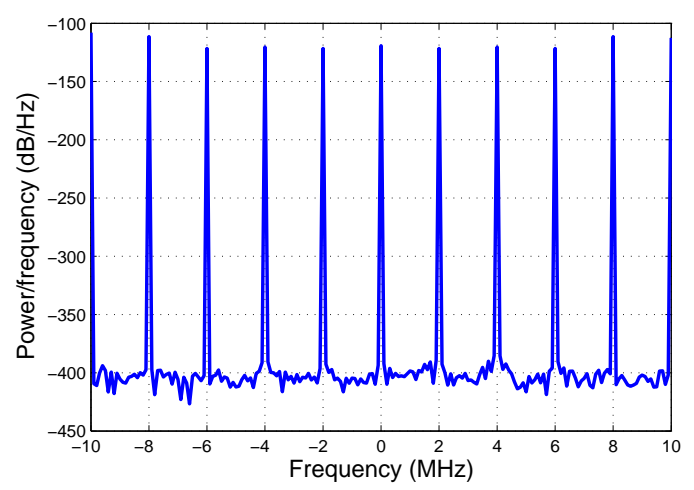

(a)

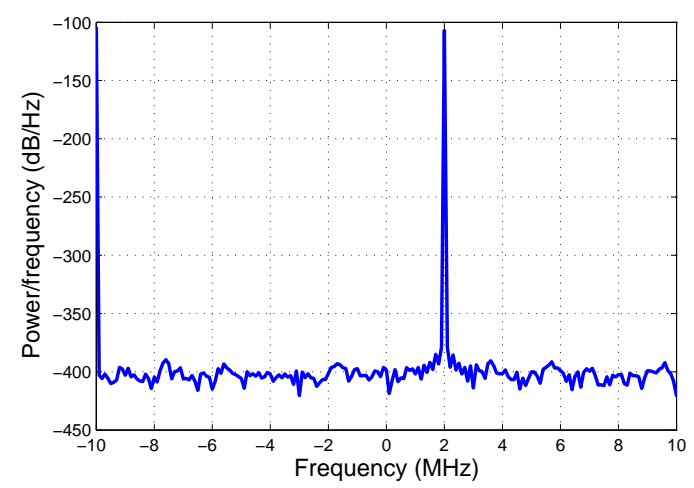

(b)

Fig. 2. Spectrum of optical signals which are phase modulated (simulated): 2(a): modulated by a sinusoidal signal $2 \pi \sin \left(2 \pi f_{\text {OFS }} t\right) ; 2(\mathrm{~b})$ modulated by a sawtooth signal $2 \pi\left[t f_{\text {OFS }}-\right.$ floor $\left.\left(t f_{\text {OFS }}\right)\right]$. Frequency 0 stands for the center frequency of the signal before modulation. $f_{\mathrm{OFS}}=2 \mathrm{MHz}$.

frequency shift of $f_{\text {OFS }}$. However, due to the bandwidth limit of the phase modulator and modulator driver, the fall time of the sawtooth signal can not be infinitely small [14]. Many sidebands are thus not totally suppressed, which affects the LDV's ability to recover the vibration velocities. To suppress these useless sidebands, we need to ensure that the fall-time is small enough, which implies that the modulator driver and the modulator need to have a much larger bandwidth than $f_{\text {OFS }}$. Most of these sidebands can also be suppressed later in the demodulator with filters, except the -1st order sideband which coincides with the 1st order harmonic. We can estimate the phase error caused by the influence of the -1st order harmonic. When the -1st order harmonic is not negligible, the demodulated phase change can be expressed as

$$
\phi_{D}^{\prime}(t)=\arctan \left[\frac{\sin 2 \phi_{D}(t)}{\cos 2 \phi_{D}(t)+\sqrt{r}}\right]-\phi_{D}(t) .
$$

where $r=P_{-1} / P_{1}$ is the power ratio between the -1 st and 1 st harmonic sidebands, and $\phi_{D}(t)$ is the actual phase change. If the ratio $r$ is quite small, the phase error $e(t)=\phi_{D}^{\prime}(t)-\phi_{D}(t)$ can be approximated as

$$
e(t) \approx-\sqrt{r} \sin 2 \phi_{D}(t) .
$$

According to equation (3), the frequency deviation is vibrating between 0 and $2 \sqrt{r} f_{D}(t)$. 


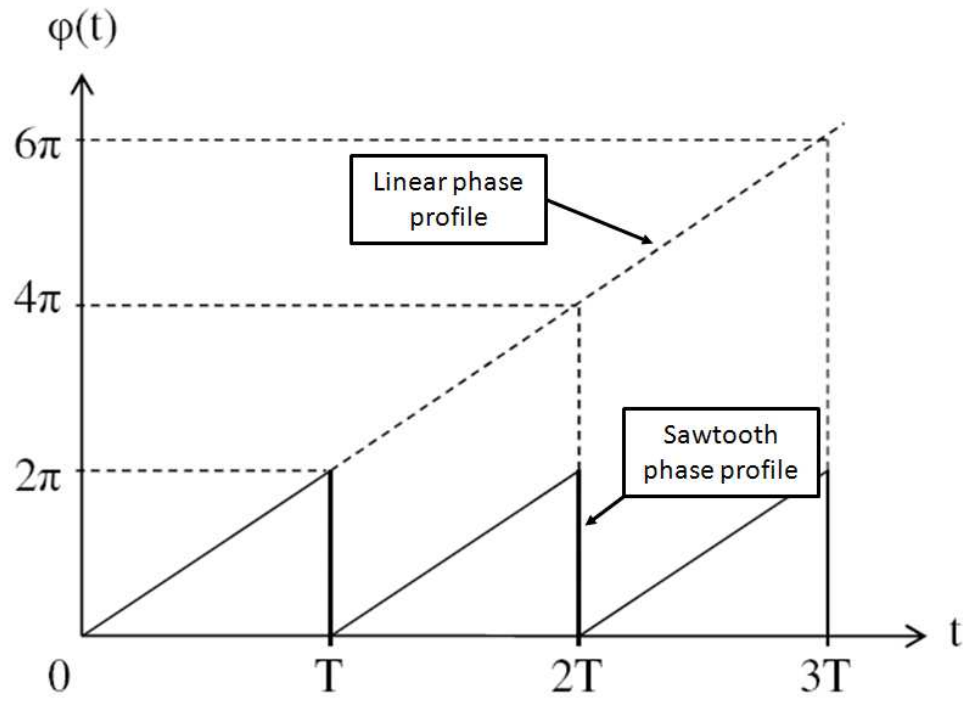

Fig. 3. The phase profile in time domain used for serrodyne optical frequency shift. $\mathrm{T}=1 / f_{\mathrm{OFS}}$.

\section{Experimental setup}

We built a serrodyne LDV based on a single-mode fiber system, the schematic configuration of which is illustrated in figure 4 .

The laser source is a distributed feedback (DFB) laser at $1550 \mathrm{~nm}$, with $3 \mathrm{~dB}$ linewidth of around $2 \mathrm{MHz}$. An isolator (not shown in the figure) was connected after the laser to avoid optical reflections to the laser. In our setup, the relative intensity noise (RIN) caused by the laser driver dominated over other noise sources when the optical power incident on the detector is higher than $100 \mathrm{nW}$. If this power was lower than $100 \mathrm{nW}$, the noise from the ADC became dominant. The demodulated signal would thus be strongly distorted due to the large relative noise. To avoid the noise problem in the ADC dominating domain, we needed to improve the reflection of the measurement signal from the moving object. A focuser was used to focus the laser beam onto one small spot on the vibrating surface. We also attached a retroreflective film on top of the moving surface, which improved the power of reflection by more than $10 \mathrm{~dB}$. When RIN dominates, the signal-to-noise ratio (SNR) can be written as

$$
\mathrm{SNR} \propto \frac{\sqrt{\alpha P_{m} P_{r}}}{\alpha P_{m}+P_{r}}
$$

where $P_{m}$ and $P_{r}$ are the powers of the measurement and reference signals just after the first optical splitter, and $\alpha$ is the optical loss of the measurement signal after scattering. The 


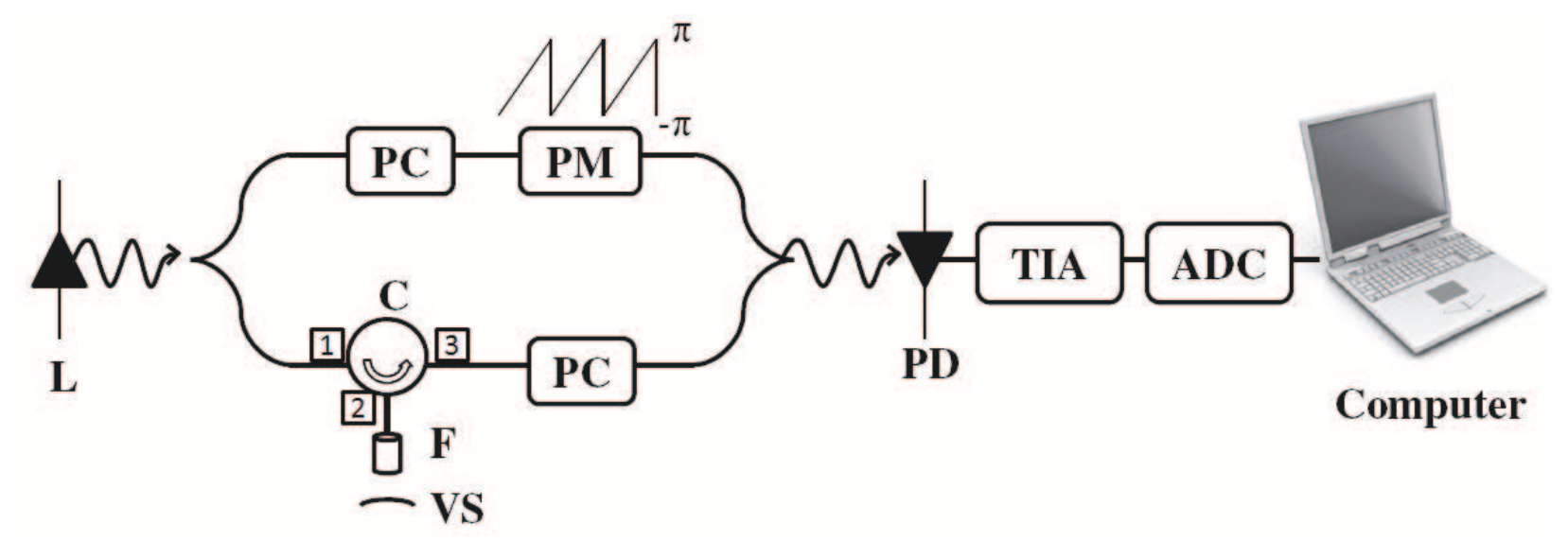

Fig. 4. Schematic configuration of the experimental set-up. In the figure, $\mathbf{L}$ stands for the laser, $\mathbf{C}$ stands for the circulator, $\mathbf{F}$ stands for the focuser, VS stands for the vibrating surface, PM stands for the phase modulator, PC stands for the polarization controller, PD stands for photo-detector, TIA stands for transimpedance amplifier, ADC stands for analog-to-digital converter.

highest SNR is obtained when $\alpha P_{m}=P_{r}$. Therefore, we used an asymmetric optical coupler to compensate the loss in the measurement arm.

The phase modulator used as the serrodyne OFS was situated in the reference arm. In the measurement arm, light goes to the vibrating surface (a moving membrane of a loudspeaker) through a circulator and focuser (the numerical aperture is 0.1). However, a small fraction of light (around $-50 \mathrm{~dB}$ ) went directly from port 1 to port 3 of the circulator, and the surface of focusers also introduced reflections. These signals do not take the useful velocity information of the vibrating surface and could deteriorate the results of demodulation.

This device is polarization sensitive, several polarization controllers are inserted to align the polarisation of measurement and reference arms. The optical path length difference of the measurement and reference arms were set to be smaller than the coherence length of the laser so that the signals from those two arms are still coherent with each other.

In the $3 \mathrm{~dB}$ optical coupler, the beams from both arms were recombined and sent to an InGaAs photodiode. The photocurrent was converted to a voltage signal by a homemade transimpedance amplifier (TIA). In the TIA, we filtered away the lower frequency signals as discussed in section 2. Then the signal is digitalized with a data acquisition card (NI USB-6218). The demodulation procedure discussed in section 2 is realized in MATLAB. 


\section{Measurement results and discussions}

With the circulator, we measured the reflected power from the moving surface with a powermeter. The optical power of the reflected signal was measured to be $20 \mathrm{~dB}$ less than the power before the signal was sent out. However, it was still $30 \mathrm{~dB}$ above the spurious reflection from the circulator and focuser. According to the discussion in the last section, in order to have a good SNR, we used a 99:1 optical coupler to split the light asymmetrically (99\% of light was sent to the measurement arm) to compensate the $20 \mathrm{~dB}$ optical loss in the measurement arm. In our experiment, we limit the power of the laser to $50 \mu \mathrm{W}$ and got $200 \mathrm{nW}$ optical power on the detector. For a laser power above $50 \mu \mathrm{W}$, we could obtain even better demodulation results from our LDV setups. An SNR of $40 \mathrm{~dB}$ was measured.

We used a function generator to create the sawtooth phase profile. Because of the linear relation between the phase and voltage in the electro-optic effect, we directly provided a sawtooth voltage to the phase modulator. When the peak-to-peak amplitude of the sawtooth voltage was $7.08 \mathrm{~V}$, we obtained a good suppression of the useless sidebands. The suppression ratio of the -1st order mode could be estimated by the power ratio of the 3rd and 1st order harmonics, since the spectrum of the serrodyne OFS is rather symmetric around the 1st order sideband [14]. The ratio was around $-35 \mathrm{~dB}$, and the corresponding phase error was smaller than $5 \%$ according to equation (11). The limited suppression ratio was mainly caused by the imperfect polarization input to the phase modulator.

We then measured the sound vibrations at several different frequencies (between $300 \mathrm{~Hz}$ and $6 \mathrm{kHz}$ ) produced by the membrane of a loudspeaker. The frequency shift $f_{\mathrm{OFS}}$ in the reference arm was set at $24 \mathrm{kHz}$. A digital bandpass filter with a center frequency of $24 \mathrm{kHz}$ and a bandwidth of $12 \mathrm{kHz}$ was used to filter away the low frequency noise and high order harmonics.

We compared our measurements with the results obtained from a commercial LDV instrument (Polytec OFV-5000), which had a higher accuracy. We pointed our fiber based LDV and the Polytec LDV to the same spot on the membrane, and measured the vibrations for the same duration. Two measurement results are shown in figure 5. It turns out that the vibration frequencies measured by both devices match well, while the vibration amplitudes are rather different (ratio $\chi$ is 0.76). This difference may be caused by the mismatch of the measuring positions for the two devices.

We defined a figure of resemblance (FOR) as

$$
\mathrm{FOR}=1-\frac{\operatorname{RMS}\left(v_{\mathrm{P}}-\chi v_{f}\right)}{\operatorname{RMS}\left(v_{\mathrm{P}}\right)},
$$

with $v_{\mathrm{P}}$ and $v_{f}$ the vibration speed measured with the Polytec LDV and our fibre LDV respectively. The calculated FOR for different vibration frequencies $f_{v i b}(t)$ and peak velocities of the membrane of the loudspeaker are shown in figure 6(a). 


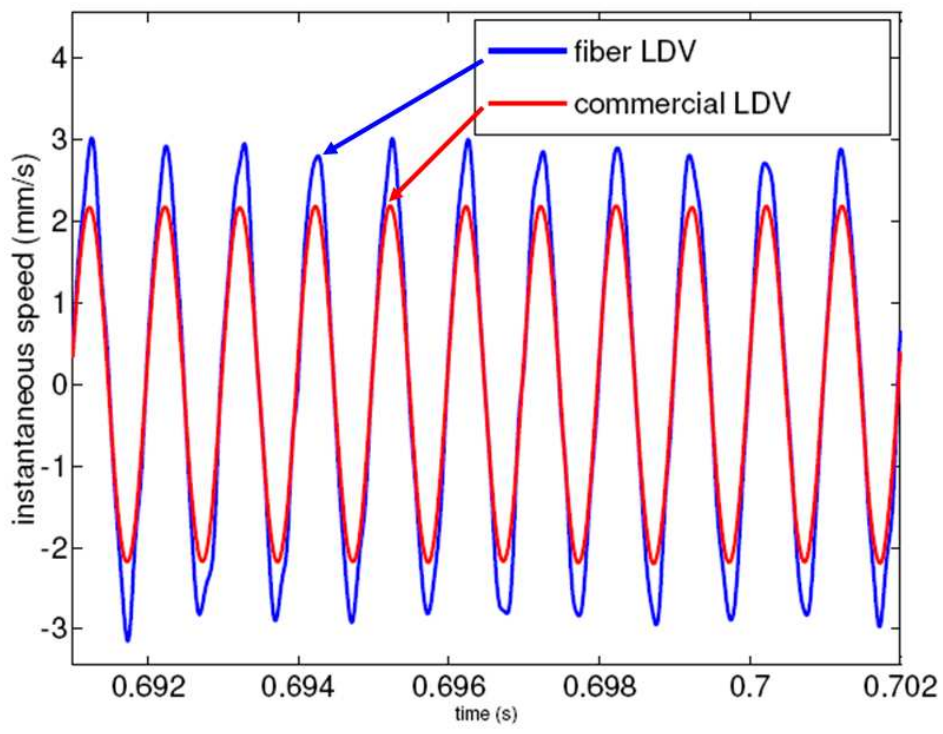

Fig. 5. Comparison of the demodulated results between Polytec LDV and fiber-based serrodyne LDV.

It can be seen that the trends for all vibration frequencies are the same. At low peak velocities (around $100 \mu \mathrm{m} / \mathrm{s}$ ), the FOR is rather low. That is because the phase variations in these situations were so small that they were strongly distorted by the phase noise. When the peak velocity surpass $300 \mu \mathrm{m} / \mathrm{s}$, the FOR is higher than $60 \%$. For peak velocity higher than $800 \mu \mathrm{m} / \mathrm{s}$, the FOR exceeds 80\%. However, at even higher speeds the FOR saturates and even drops slightly. This is because the bandwidth of our signal became larger than the bandwidth $(12 \mathrm{kHz})$ around $f_{\mathrm{OFS}}$. Information would then be lost after filtering, resulting in distortions. We increased the bandpass filter bandwidth around $f_{\mathrm{OFS}}$, and the FOR figure is as in figure 6(b). The dropping part in figure 6(a) disappears. But noise introduced by the larger filter bandwidth deteriorated the demodulation and decreased the FOR for low speeds vibrations.

Our measured vibrations with sound frequencies starting from around $1000 \mu \mathrm{m} / \mathrm{s}$ is relatively accurate, with a FOR larger than $80 \%$. This corresponds with a peak displacement in the order of $100 \mathrm{~nm}$.

\section{Conclusions}

We proposed and demonstrated a serrodyne optical frequency shifting (SFS) technique as a candidate for the frequency shifter in the miniaturized heterodyne LDV. The theoretical analysis shown that an ideal SFS can be a perfect single sideband frequency shifter, but in 


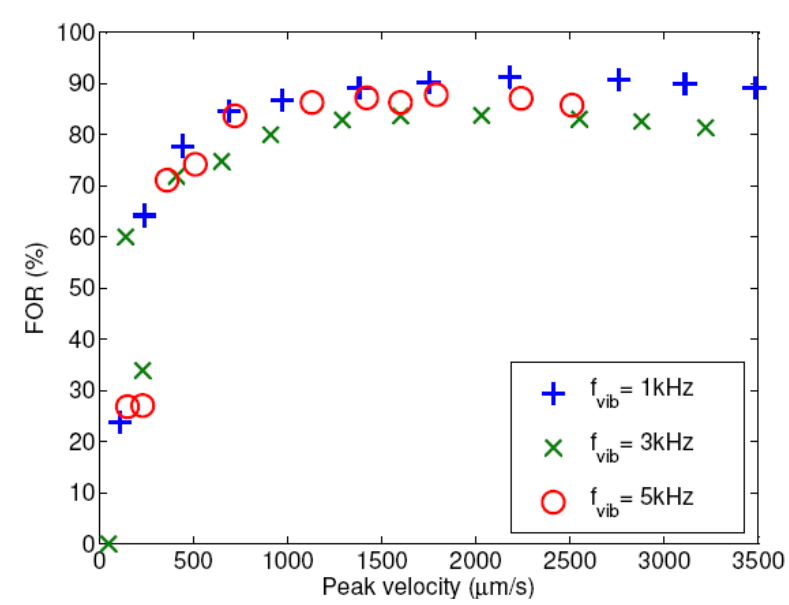

(a)

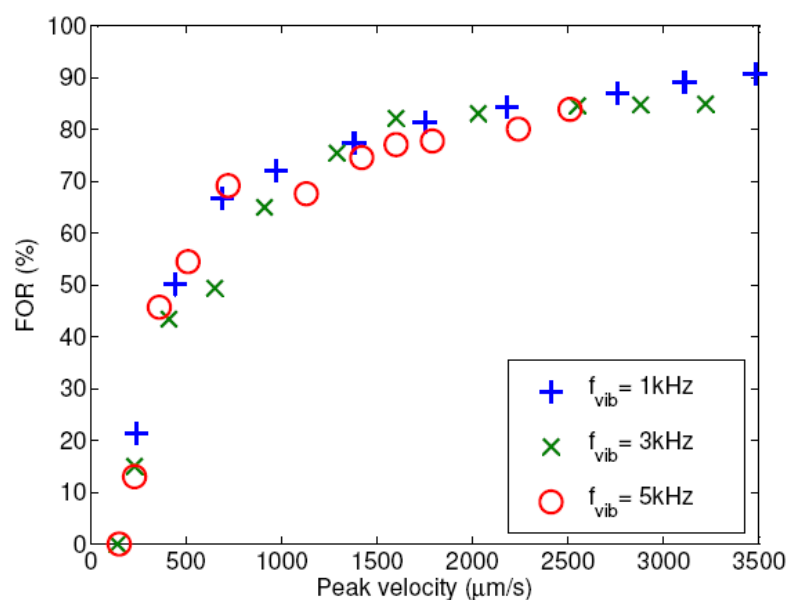

(b)

Fig. 6. Figure of resemblance (FOR) for different vibration frequencies and speeds with difference bandwidths of bandpass filters. 6(a): bandwidth of $12 \mathrm{kHz}$. 6(b): bandwidth of $24 \mathrm{kHz}$.

real world it was limited by the bandwidth of the modulator and modulator driver. The -1st order harmonic influenced the demodulation results and should be highly suppressed in the OFS. A fibre-based LDV system with telecommunication components (lasers and detectors) was built to make the proof-of-principle for LDVs working with SFS. While limiting the power of our laser (at $1550 \mathrm{~nm}$ ) to $50 \mu \mathrm{W}$, our measurements on audio frequency vibrations with submicron displacements were still acceptable. The optical frequency shift used in the reference arm was $24 \mathrm{kHz}$.

\section{Acknowledgements}

The authors acknowledge the Research Foundation - Flanders (FWO) through contract 3G003410 for partial support. They appreciate Pieter Wiskerke, Gerald Dumm, and Patrik Kennes (Cochlear). They also thank prof. Joris Peeters, prof. Patrick Segers, dr. Danae Delbeke, ir. Gunay Yurtsever, and ing. Jeroen Allaert for discussions about this work.

\section{References}

1. A. T. Waz, P. R. Kaczmarek, and K. M. Abramski, "Laser-fibre vibrometry at 1550 nm," Measurement Science and Technology 20, 105301 (2009).

2. P. Gren, K. Tatar, J. Granström, N.-E. Molin, and E. V. Jansson, "Laser vibrometry measurements of vibration and sound fields of a bowed violin," Meas. Sci. Technol. 17, 
635-644 (2006).

3. L. Jacquin, D. Fabre, D. Sipp, V. Theofilis, and H. Vollmers, "Instability and unsteadiness of aircraft wake vortices," Aerospace Science and Technology 7, 577-593 (2003).

4. K. R. Whittemore, S. N. Merchant, B. B. Poon, and J. J. Rosowski, "A normative study of tympanic membrane motion in humans using a laser doppler vibrometer (ldv)," Hearing Research 187, 85-104 (2004).

5. N. P. Cooper, "An improved heterodyne laser interferometer for use in studies of cochlear mechanics," Journal of Neuroscience Methods 88, 93-102 (1999).

6. M. Johansmann, G. Siegmund, and M. Pineda, "Targeting the limits of laser doppler vibrometry," Proc. IDEMA 2005 pp. 1-12 (2005).

7. D. Vermeulen, S. Selvaraja, P. Verheyen, G. Lepage, W. Bogaerts, P. Absil, D. V. Thourhout, and G. Roelkens, "High-efficiency fiber-to-chip grating couplers realized using an advanced cmos-compatible silicon-on-insulator platform," Opt. Express 18, 18278-18283 (2010).

8. Z. Sheng, L. Liu, J. Brouckaert, S. He, and D. V. Thourhout, "Ingaas pin photodetectors integrated on silicon-on-insulator waveguides," Opt. Express 18, 1756-1761 (2010).

9. L. Liu, R. Kumar, K. Huybrechts, T. Spuesens, G. Roelkens, E.-J. Geluk, T. de Vries, P. Regreny, D. Van Thourhout, R. Baets, and G. Morthier, "An ultra-small, low-power, all-optical flip-flop memory on a silicon chip," NATURE PHOTONICS 4, 182-187 (2010).

10. J. V. Campenhout, P. R. Romeo, P. Regreny, C. Seassal, D. V. Thourhout, S. Verstuyft, L. D. Cioccio, J.-M. Fedeli, C. Lagahe, and R. Baets, "Electrically pumped inp-based microdisk lasers integrated with a nanophotonic silicon-on-insulator waveguide circuit," Opt. Express 15, 6744-6749 (2007).

11. J. Brouckaert, G. Roelkens, D. Van Thourhout, and R. Baets, "Compact inalasingaas metalsemiconductormetal photodetectors integrated on silicon-on-insulator waveguides," Photonics Technology Letters, IEEE pp. $1484-1486$.

12. R. C. Cumming, "The serrodyne frequency translator," Proceedings of the Institute of Radio Engineers 45, 175-186 (1957).

13. I. Y. Poberezhskiy, B. Bortnik, J. Chou, B. Jalali, and H. R. Fetterman, "Serrodyne frequency translation of continuous optical signals using ultrawide-band electrical sawtooth waveforms," IEEE J. Quantum Electron. 41, 1533-1539 (2005).

14. L. M. Johnson and C. H. Cox, "Serrodyne optical frequency translation with high sideband suppression," J. Lightwave Technol. 6, 109-112 (1988). 